\title{
Indication for endoscopic treatment based on the risk of lymph node metastasis in patients with ulcerative-type early gastric cancer
}

\author{
Ji Won Kim${ }^{1}$, Hyuk Lee ${ }^{1}$, Byung-Hoon Min¹, Jun Haeng Lee ${ }^{1}$, Kyoung-Mee Kim², \\ Ji Yeong An ${ }^{3}$, Min Gew Choi ${ }^{3}$, Jun Ho Lee ${ }^{3}$, Tae Sung Sohn ${ }^{3}$, Jae Moon Bae ${ }^{3}$, Jae J \\ $\mathrm{Kim}^{1}$ and Sung $\mathrm{Kim}^{3}$ \\ ${ }^{1}$ Department of Medicine, Samsung Medical Center, Sungkyunkwan University School of Medicine, Seoul, South Korea \\ ${ }^{2}$ Department of Pathology, Samsung Medical Center, Sungkyunkwan University School of Medicine, Seoul, South Korea \\ ${ }^{3}$ Department of Surgery, Samsung Medical Center, Sungkyunkwan University School of Medicine, Seoul, South Korea \\ Correspondence to: Hyuk Lee, email: leehyuk@skku.edu \\ Tae Sung Sohn, email: tssohn@skku.edu
}

Keywords: ulcerative early gastric cancer; lymph node metastasis; submucosal invasion; tumor size

Received: July 26, 2017 Accepted: November 17, $2017 \quad$ Published: January 10, 2018

Copyright: Kim et al. This is an open-access article distributed under the terms of the Creative Commons Attribution License 3.0 (CC BY 3.0), which permits unrestricted use, distribution, and reproduction in any medium, provided the original author and source are credited.

\section{ABSTRACT}

Background: There is little evidence regarding the appropriate therapeutic modalities for ulcerative-type early gastric cancer (EGC) because the risks and implications of lymph node metastasis (LNM) are unclear. In this study, the indication for endoscopic submucosal dissection (ESD) was investigated in patients with ulcerative-type EGC.

Results: The overall LNM rate was $15.1 \%, 0 \%$ for mucosa-confined lesions, and $\mathbf{2 8 . 2 \%}$ for submucosa-infiltrating lesions. In the multivariate analysis, only lymphovascular invasion $(P<\mathbf{0 . 0 0 1})$ was significantly associated with LNM. Among patients with minute submucosal invasion and no lymphovascular invasion, LNM was only observed in patients with tumor sizes $>2.0 \mathrm{~cm}$.

Materials and Methods: We retrospectively analyzed 192 patients with ulcerativetype EGC who underwent radical gastrectomy with D2 lymph node dissection. The LNM risk factors were evaluated using a multivariate logistic regression analysis.

Conclusions: Because the LNM risks are negligible, curative ESD could be considered in patients with ulcerative EGC that is confined to the mucosa and histologically differentiated, irrespective of tumor size. Additionally, ESD can be attempted in patients with ulcerative EGC with minute submucosal invasion and a tumor size $\leq 2.0 \mathrm{~cm}$.

\section{INTRODUCTION}

Endoscopic submucosal dissection (ESD) is an effective alternative to surgical treatment for patients with early gastric cancer (EGC) and minimal risks of developing regional lymph node metastasis (LNM) [1]. ESD is less invasive and more effective than surgery [2]. Because ESD should be applied in patients with EGC without LNM, it is important to evaluate the risk of LNM in patients who undergo EGC [3-5]. Therefore, the traditionally accepted indication for ESD has been limited to differentiated intramucosal cancer that is less than $20 \mathrm{~mm}$ in diameter, without ulceration. However, studies have suggested that selected patients with EGC can also be considered candidates for ESD if they have low risks of LNM [6-8]. Thus, the indication for ESD has been expanded [9-11]. Notably, for example, the Japanese Gastric Cancer Association has recommended an extended indication for ESD that includes differentiated intramucosal cancer $\leq 3 \mathrm{~cm}$ with ulceration [12]. This indication originated from a few reports that attempted to evaluate the risk factors of LNM in patients with ulcerative-type EGC. However, evidence about the risk of LNM in patients with ulcerative ECG has been scarce 
because relatively small numbers of patients are diagnosed with this kind of cancer [13]. Furthermore, in the majority of patients with type III gastric cancer, submucosal invasion seems to be associated with LNM. Indeed, the risk of LNM has seldom been evaluated in patients who have ulcerative intramucosal gastric cancer.

Therefore, we performed this study to identify the factors that are related to LNM in patients with ulcerative EGC and to establish a suitable subgroup of patients with ulcerative EGC for whom ESD could be performed.

\section{RESULTS}

\section{Patient characteristics}

Prospectively collected data showed that 8,791 patients were diagnosed with EGC and underwent gastrectomy with lymphadenectomy. Among the total number of EGC patients, 490 had ulcerative-type EGC (5.6\% of all EGC lesions), including 86 with pure ulcerative lesions $(0.98 \%$ of all EGC lesions). After excluding patients with tumors with undifferentiated histology, we confirmed that 192 patients $(2.2 \%$ of all EGC cases) had ulcerative-type, differentiated EGC. Of the 192 patients with ulcerative, differentiated-type EGC, $164(85.4 \%)$ were men and $28(14.6 \%)$ were women. Their mean age was 58.2 years (range: $26-88$ ). The mean number of lymph nodes that were removed was 37.3 (range: 6 - 119). Of the patients, 46.4\% (89/192) had mucosal cancer and 53.5\% (103/192) had submucosal, infiltrative cancer. Regarding the submucosal (SM) categories, 46 (24.0\%), 28 (14.6\%), and 29 (15.1\%) of the lesions were SM1, SM2, and SM3, respectively. The overall rate of LNM was $15.1 \%$ (29/192). The mean number of metastatic lymph nodes per patient was 2.3 (range: 1-11). Although LNM was not observed in any patients with lesions that were confined to the mucosa, LNM was found in $29(28.2 \%)$ of the patients with lesions that had infiltrated the submucosa (Table 1).

\section{Clinicopathological characteristics related to the presence of LNM}

Table 2 compares the clinicopathological features of patients with ulcerative EGCs according to whether LNM was present. Submucosal invasion $(P<0.001)$ and the presence of lymphovascular invasion $(P<0.001)$ were significantly associated with LNM. Especially, no patient had LNM and intramucosal cancer. Regarding submucosal, invasive cancer, we noted that there was a high incidence of LNM in the SM2 and SM3 subgroups. There were no significant differences between the patients with and without LNM regarding factors such as age, sex, tumor location, tumor size, or World Health Organization (WHO) histologic type.

In the univariate analysis, the depth of invasion and lymphovascular invasion were significantly associated with LNM. However, LNM was not significantly associated with the tumor size or WHO histopathologic tumor type. In the multivariate analysis, only lymphovascular invasion was significantly associated with LNM (hazard ratio: 4.1, 95\% confidence interval: 1.7-10.1) (Table 3).

To account for the small sample sizes and event rates in our study, we also applied Firth's penalized maximum likelihood estimation approach, which showed similar results to those described previously. In the multivariate analysis that used Firth's method, lymphovascular invasion was the only factor that showed a significant association with LNM. LNM was not significantly associated with the tumor size (maximal or minimal) or WHO histopathologic tumor type (Table 4).

\section{Group classification for endoscopic resection}

None of the patients with ulcerative, intramucosal cancer had LNM, regardless of their tumor sizes. Among patients with submucosal invasion less than $<500 \mu \mathrm{m}$, the mean tumor size did not differ between those who did and did not have LNM (mean \pm standard deviation: 3.97 $\pm 1.84 \mathrm{~cm}$ vs. $3.07 \pm 1.61 \mathrm{~cm}$, respectively, $P=0.150)$. In the group of patients with SM1 cancer, the smallest tumor size - in a patient with LNM-was $2.1 \mathrm{~cm}$ (Figure 1). The receiver operating characteristic curve based on the maximal tumor size is shown in Figure 2. The tumor size had an area under the curve of 0.65 (95\% confidence interval: $0.50-0.79)$, with a cut-off tumor size of $3.5 \mathrm{~cm}$. For patients with a tumor size $>3.5 \mathrm{~cm}$, the sensitivity, specificity, positive predictive value, and negative predictive value for LNM were 55.6\%, 81.1\%, 41.7\%, and $88.2 \%$, respectively. Using a tumor size of $>2.0 \mathrm{~cm}$ instead showed corresponding values of $100 \%, 24.3 \%$, $24.3 \%$, and $100 \%$, respectively.

\section{DISCUSSION}

EGC is defined as a gastric carcinoma in which tumor invasion is confined to the mucosa or submucosa, regardless of whether the patient has LNM. The 10-year survival rate of patients with EGC is $>90 \%$, and the LNM status is the most important prognostic factor [14-18]. When EGC is categorized into mucosal and submucosal cancer, the prognosis of mucosal cancer is significantly better than that of submucosal cancer because of the higher incidence of LNM in the latter category [19]. Therefore, the absolute indication of ESD for EGC is tumors that are confined to the mucosa. Because of the increased desire for minimally invasive treatment that maintains normal gastric function, and because of the technical improvements that have been achieved in endoscopic procedures, an extended indication for endoscopic resection has been proposed [20]. However, because ulcerative EGC is associated with a higher incidence of LNM, ESD has only been used in 
Table 1: The clinicopathologic characteristics of the subjects

\begin{tabular}{lc}
\hline Characteristics & Patients \\
\hline Age, yr (mean, range) & $58.2(26 \sim 88)$ \\
Sex, $\mathrm{n}(\%)$ & \\
Male & $164(85.4)$ \\
Female & $28(14.6)$ \\
Tumor location, $\mathrm{n}(\%)$ & \\
Upper & $15(7.8)$ \\
Middle & $53(27.6)$ \\
Lower & $124(64.6)$ \\
Tumor size, cm (mean \pm SD) & \\
Max & $3.26 \pm 1.59$ \\
Min & $2.18 \pm 1.18$ \\
Histologic type, $\mathrm{n}(\%)$ & \\
Well differentiated & $31(15.5)$ \\
Moderate differentiated & $169(84.5)$ \\
Depth of invasion, $\mathrm{n}(\%)$ & \\
Mucosa & $89(46.4)$ \\
Submucosa & $103(53.7)$ \\
SM1 & $46(24.0)$ \\
SM2 & $28(14.6)$ \\
SM3 & $29(15.1)$ \\
Lymphovascular invasion, $\mathrm{n}(\%)$ & \\
Absence & $153(79.7)$ \\
Presence & $39(20.3)$ \\
\hline
\end{tabular}

SD: standard deviation.

highly selective cases [21]. According to the expanded indication for ESD, endoscopic treatment has not been applicable in patients with ulcerative, intramucosal cancer greater than $3 \mathrm{~cm}$. Furthermore, ESD is not indicated for patients with ulcerative EGC with minute submucosal cancer $[6,21,22]$. However, there has not been enough evidence to reveal the clinical relevance of this guideline. Thus, we sought to expand the use of ESD to ulcerative EGC based on a retrospective examination in which we identified the predictive factors of LNM.

In the present study, the univariate analyses revealed that the depth of invasion and lymphovascular invasion were significant factors that predicted LNM. This supports the results of previous studies on EGC, which showed that an elevated incidence of LNM was significantly correlated with the depth of invasion and lymphovascular invasion [23-25]. However, LNM was not significantly associated with the tumor size or histopathologic tumor type. In the multivariate analysis, only lymphovascular invasion was significantly associated with LNM. Although the tumor size and invasion depth were not independent predictive factors, these two parameters can be considered for re- establishing the indication for endoscopic resection [26]. None of the intramucosal cancer patients in our cohort had LNM. In other words, unlike previous guidelines, our results suggest that endoscopic treatment can be attempted in patients with intramucosal cancer without regard to the tumor size. In addition, the disease can be re-classified regarding the risk of LNM in patients with submucosal, invasive cancer. Notably, among patients with ulcerative EGC and minute submucosal invasion, there were no LNMs in patients with tumor sizes less than $2 \mathrm{~cm}$. This may be an indication for endoscopic treatment of ulcerative EGC.

When deciding the treatment modalities (endoscopic resection or surgical gastrectomy), evaluating the possibility of lymph node metastasis is vital. If we encounter ulcerative-type EGC, considering the current expanded indication for ESD, an intramucosal lesion can be considered a feasible indicator of ESD, but only in lesions that are less than $3 \mathrm{~cm}$. Regarding submucosal cancer, endoscopic treatment cannot be attempted in patients with ulcerative lesions. However, there is a considerable discrepancy between the pre-ESD and 
Table 2: The clinicopathologic characteristics that were related to the presence of lymph node metastasis in patients with ulcerative early gastric cancer

\begin{tabular}{|c|c|c|c|}
\hline & \multicolumn{2}{|c|}{ Lymph node metastasis } & \multirow[b]{2}{*}{$P$ value } \\
\hline & $\begin{array}{l}\text { Negative } \\
(n=165)\end{array}$ & $\begin{array}{l}\text { Positive } \\
(n=27)\end{array}$ & \\
\hline Age, yr (mean \pm SD) & $57.7 \pm 10.8$ & $61.4 \pm 11.2$ & 0.918 \\
\hline Sex, $n(\%)$ & & & 0.225 \\
\hline Male & $149(88.2)$ & $25(80.6)$ & \\
\hline Female & $20(11.8)$ & $6(19.3)$ & \\
\hline Tumor location, n(\%) & & & 0.112 \\
\hline Upper & $11(6.5)$ & $2(6.5)$ & \\
\hline Middle & $51(30.2)$ & $4(12.9)$ & \\
\hline Lower & $107(63.3)$ & $25(80.6)$ & \\
\hline Gross type, $\mathrm{n}(\%)$ & & & 0.527 \\
\hline Pure ulcerative & $33(20.0)$ & $4(14.8)$ & \\
\hline Mixed ulcerative & $132(80.0)$ & $23(85.2)$ & \\
\hline \multicolumn{4}{|l|}{ Tumor size, $\mathrm{mm}($ mean $\pm \mathrm{SD})$} \\
\hline Max & $3.21 \pm 1.54$ & $3.50 \pm 1.85$ & 0.105 \\
\hline Min & $2.13 \pm 1.18$ & $2.48 \pm 1.16$ & 0.944 \\
\hline \multicolumn{4}{|l|}{ Histologic type, $\mathrm{n}(\%)$} \\
\hline Well differentiated & $24(17.0)$ & $3(11.1)$ & 0.443 \\
\hline Moderate differentiated & $137(83.0)$ & $28(88.9)$ & \\
\hline Depth of invasion, $\mathrm{n}(\%)$ & & & $<0.001$ \\
\hline Mucosa & $89(53.9)$ & 0 & \\
\hline \multicolumn{4}{|l|}{ Submucosa } \\
\hline SM1 & $37(22.4)$ & $9(19.6)$ & \\
\hline SM2 & $16(9.7)$ & $12(42.9)$ & \\
\hline SM3 & $23(13.9)$ & $6(20.7)$ & \\
\hline Lymphovascular invasion, $\mathrm{n}(\%)$ & & & $<0.001$ \\
\hline Absence & $140(84.8)$ & $12(44.4)$ & \\
\hline Presence & $25(15.2)$ & $15(55.6)$ & \\
\hline
\end{tabular}

SD: standard deviation.

post-ESD pathology. Hence, in real practice, endoscopic resection is frequently performed in patients with an ulcerative lesion because of ambiguity about the size or depth. Usually, after the final pathologic interpretation of a specimen that is taken during endoscopic resection, additional surgical resection is conducted in selected cases, beyond the expanded indication. Finally, predicting lymph node metastasis using a pathologic specimen after endoscopic resection is needed for the optimal section of additional surgical candidates. However, there is insufficient evidence regarding lymph node metastasis in patients with ulcerative, differentiated EGC because a small number of lesions fall into this category. In our large-scale study, there was a definite difference from the known expanded indication. We found that endoscopic resection can be applied in patients with intramucosal, ulcerative EGC, regardless of the tumor size. Additionally, a minute submucosal ulcerative-type cancer lesion that is less than $2 \mathrm{~cm}$ in size that does not fall under the existing expanded indication can be considered in our modified 
Table 3: The risk factors of lymph node metastasis in patients with ulcerative-type EGC

\begin{tabular}{|c|c|c|c|c|c|c|}
\hline & \multicolumn{3}{|c|}{ Univariate analysis } & \multicolumn{3}{|c|}{ Multivariate analysis } \\
\hline & OR & $95 \% \mathrm{CI}$ & $P$ value & OR & $95 \% \mathrm{CI}$ & $P$ value \\
\hline \multicolumn{7}{|l|}{ Tumor size } \\
\hline Max & 1.09 & $0.86-1.37$ & 0.485 & 0.88 & $0.53-1.38$ & 0.598 \\
\hline Min & 1.24 & $0.92-1.68$ & 0.158 & 1.34 & $0.75-2.42$ & 0.274 \\
\hline \multicolumn{7}{|l|}{ Histologic type } \\
\hline Well differentiated & 1.00 & & & & & \\
\hline Moderate differentiated & 1.85 & $0.53-6.52$ & 0.336 & 2.08 & $0.54-7.99$ & 0.285 \\
\hline \multicolumn{7}{|l|}{ Depth of invasion } \\
\hline Mucosa & 1.00 & & & & & \\
\hline Submucosa & 16.04 & $2.16-28.01$ & 0.047 & 7.26 & $0.91-9.39$ & 0.164 \\
\hline \multicolumn{7}{|l|}{ Lymphovascular invasion } \\
\hline Absence & 1.00 & & & & & \\
\hline Presence & 6.39 & $2.82-14.48$ & 0.0001 & 7.13 & $3.03-16.79$ & 0.0001 \\
\hline
\end{tabular}

ECG: early gastric cancer; OR: odds ratio; CI: confidence interval.

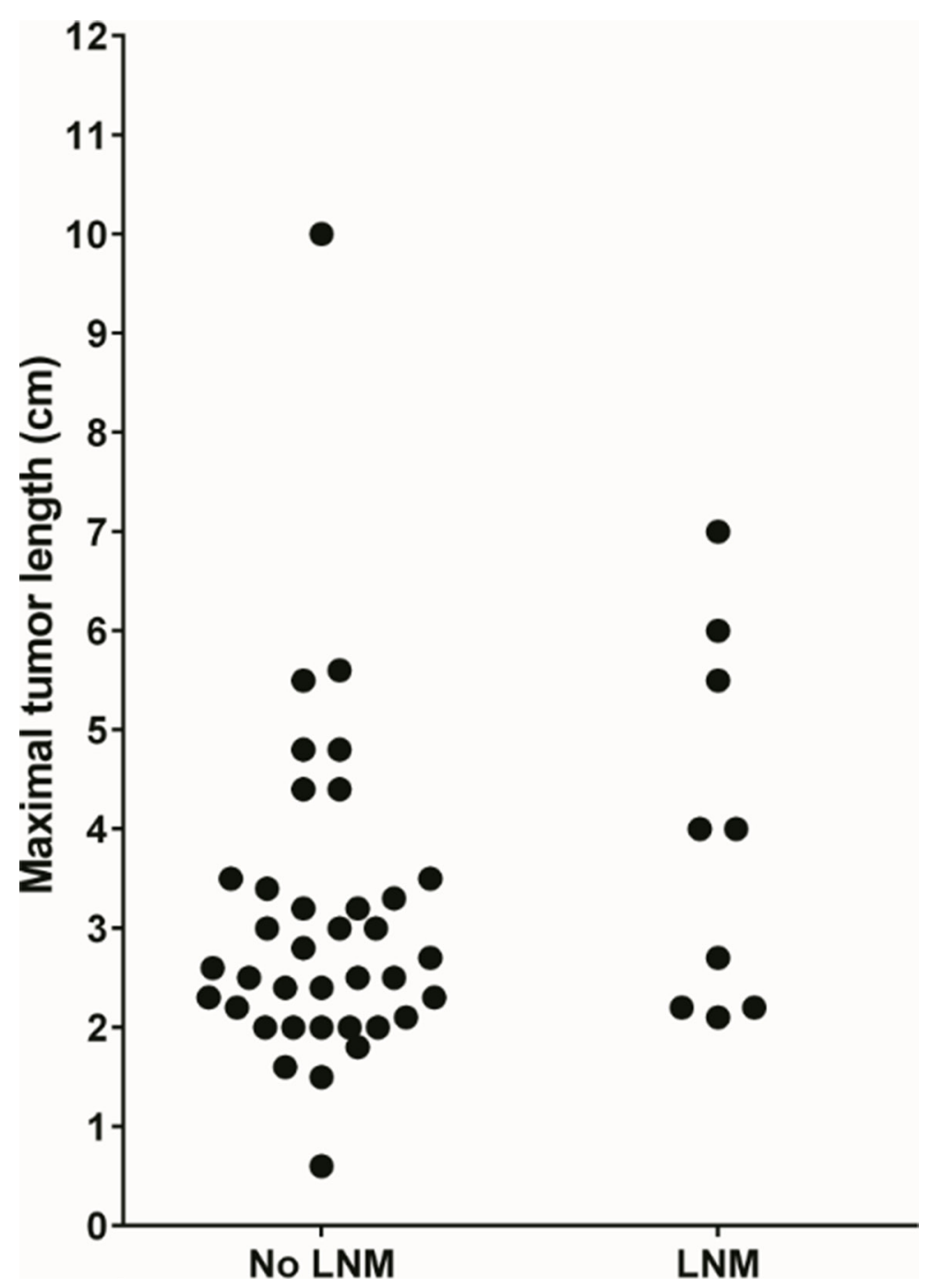

Figure 1: Distribution of the tumor size according to lymph node metastasis in patients with ulcerative early gastric cancer with minute submucosal invasion. 


\begin{tabular}{|c|c|c|c|}
\hline & \multicolumn{3}{|c|}{ Multivariable Firth's method } \\
\hline & Odd ratio & $95 \%$ confidence interval & $P$ value \\
\hline \multicolumn{4}{|l|}{ Tumor size } \\
\hline Max & 2.664 & $0.081-8.862$ & 0.110 \\
\hline Min & 1.571 & $0.617-3.998$ & 0.344 \\
\hline Histologic type & 2.116 & $0.561-7.980$ & 0.269 \\
\hline Depth of invasion & 7.949 & $0.479-131.87$ & 0.148 \\
\hline Lymphovacular invasion & 5.612 & $2.332-13.506$ & 0.0001 \\
\hline
\end{tabular}

indication. Namely, the risk of LNM in patients with ulcerative-type, differentiated EGC might have been overestimated in past studies. Table 5 shows the modified indication for endoscopic resection in patients with ulcerative-type EGC.

To our knowledge, this study is the first to investigate the risk of LNM in patients with ulcerative EGC regarding endoscopic treatment. Moreover, a strength of this study was that we analyzed a large cohort of EGC patients, even though the data were collected from a single center. Our study also has a weakness. Although the majority of patients received D2 or D2+ lymphadenectomy, this study was not a randomized controlled study, and the extent of lymphadenectomy was not equal in all patients. Therefore, nodal metastases could have been underestimated in some patients, leading to selection bias.

\section{Tumor size}

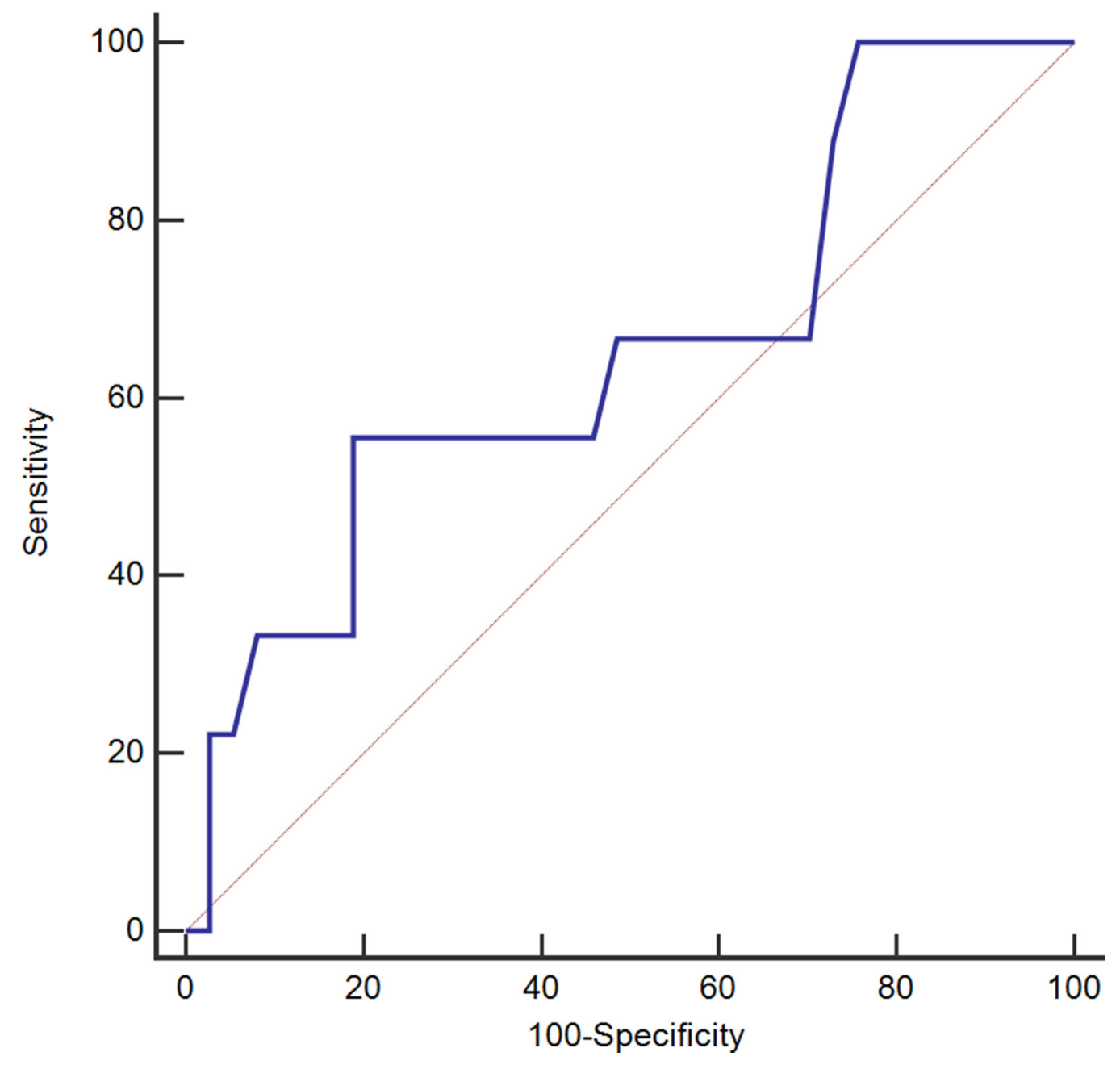

Figure 2: The receiver operating characteristic curve for predictions of lymph node metastasis, based on the tumor size in patients with ulcerative early gastric cancer with minute submucosal invasion. 
Table 5: The modified criteria for endoscopic treatment in patients with ulcerative-type, differentiated EGC

\begin{tabular}{ccc}
\hline \multirow{2}{*}{ Histology } & \multicolumn{2}{c}{ Ulcerative-type } \\
\cline { 2 - 3 } & & Depth \\
\cline { 2 - 3 } & Mucossl cancer & Submucosal cancer $<\mathbf{5 0 0} \boldsymbol{\mu m}$ \\
\hline Differentiated & All size & $<2.1 \mathrm{~cm}$ \\
\hline
\end{tabular}

EGC: early gastric cancer.

In conclusion, because the risk of LNM appears to be negligible, curative ESD could be considered for patients with ulcerative EGC that is confined to the mucosa and has a well-differentiated histology, irrespective of the tumor size. In addition, endoscopic treatment can be attempted in patients with ulcerative EGC and minute submucosal invasion and a tumor size less than $2 \mathrm{~cm}$. This finding should be confirmed with additional data from other centers, in studies that focus on LNM after ESD in patients with ulcerative-type EGC.

\section{MATERIALS AND METHODS}

\section{Ethical considerations}

This study was approved by the Institutional Review Board of Samsung Medical Center.

\section{Study population}

Patients with pathologically diagnosed T1 (confined to the mucosa and submucosa) gastric cancer were selected from a prospective database of the Department of Surgery, Samsung Medical Center, between January 2003 and December 2014. We included patients with newly diagnosed EGC without previous gastric cancer treatment. From the patients who met these criteria, we selected those with type III lesions with an ulcerative appearance, based on definitive and consistent reviews of pathologic reports. The macroscopic classification was decided according to the criteria of the Japanese Society of Gastroenterological Endoscopy [27]. An experienced pathologist reviewed all sections of formalin-fixed and paraffin-embedded specimens that were stained with hematoxylin and eosin. The microscopic features were recorded according to the criteria of the Japanese Research Society for Gastric Cancer classification [28].

We excluded patients with cancers that had poorly differentiated or signet ring cell histology. After applying the inclusion and exclusion criteria, in this study, we included 192 patients with differentiated type III T1 gastric cancer that was confined in the mucosal and submucosal layers. We collected patient data on age, sex, presence of lymphovascular invasion, tumor size and location, differentiation stage, depth of invasion, whether R0 resection was achieved, presence of a synchronous tumor, number of lymph nodes that were dissected, and Lauren classification.

\section{Surgical procedure}

Distal, proximal, or total gastrectomy was performed according to the tumor's location. The extent of lymph node dissection (D1+, D2, or D2+) was based on the Japanese Gastric Cancer Treatment Guidelines [28]. D2 or D2+ was performed whenever nodal involvement was suspected. For reconstruction after gastrectomy, Roux-en-Y reconstruction was preferentially performed after total gastrectomy, and esophagogastrostomy was performed after proximal gastrectomy.

\section{Histopathologic evaluation}

All surgical specimens were routinely fixed in $10 \%$ formalin and were serially sectioned at $5-\mathrm{mm}$ intervals, embedded in paraffin blocks, and stained with hematoxylin and eosin. The depth of the tumor's invasion was then evaluated based on the lymphovascular involvement and degree of differentiation. Lymphatic vessel invasion was defined as the presence of neoplastic cell emboli within spaces that were surrounded by a clearly visualized endothelial lining. Vascular invasion was identified by the presence of additional fibrin clots, erythrocytes, or both within an endothelial-lined space, without erythrocyte extravasation into the surrounding tissue; or by evidence of neoplastic cells within a smooth muscle cell-lined space. A tumor was classified as either lymphatic vessel invasion-negative or vascular invasionnegative if an examination of the entire periphery of the tumor sample on the slides did not reveal tumor cells within the endothelium-lined spaces [29]. The lymph nodes were cut into two pieces, and the cut surfaces were examined to determine the status of the lymph nodes. LNM was identified using hematoxylin and eosin staining. The tumors were classified according to the American Joint Committee on Cancer Staging Manual (7th edition) [30]. Submucosal invasion was divided into the upper (SM1: <500 $\mu \mathrm{m}$ from the muscularis mucosae), middle (SM2), and lower third (SM3) invasion. R0 resection was defined as resection of the tumor without histologic evidence of cancer on the lateral or vertical margins. 


\section{Statistical analysis}

The primary endpoint was LNM. Univariate analyses were performed using the chi-squared test or Fisher's exact test for categorical variables, and Student's $t$-test was used for continuous variables. To identify the risk factors of LNM in patients with ulcerative EGC, we used a forward, stepwise, multiple logistic regression model, including the variables that had been statistically significant $(P<0.05)$ in the univariate analyses. $P$-values $<0.05$ indicated statistical significance. SPSS version 18.0 for Windows (SPSS Inc., Chicago, IL, USA) was used for the statistical analyses. Because logistic regression was used for rare events, we additionally applied Firth's penalized maximum likelihood estimation method to explore the validity of the tumor size criterion for predicting LNM.

\section{CONFLICTS OF INTEREST}

None.

\section{GRANT SUPPORT}

None.

\section{REFERENCES}

1. Oka S, Tanaka S, Kaneko I, Mouri R, Hirata M, Kawamura T, Yoshihara M, Chayama K. Advantage of endoscopic submucosal dissection compared with EMR for early gastric cancer. Gastrointest Endosc. 2006; 64:877-883.

2. Pyo JH, Lee H, Min BH, Lee JH, Choi MG, Lee JH, Sohn TS, Bae JM, Kim KM, Ahn JH, Carriere KC, Kim JJ, Kim S. Long-Term Outcome of Endoscopic Resection vs. Surgery for Early Gastric Cancer: A Non-inferiorityMatched Cohort Study. Am J Gastroenterol. 2016; 111:240-249.

3. Kim SG. Endoscopic treatment for early gastric cancer. J Gastric Cancer. 2011; 11:146-154.

4. Guo CG, Zhao DB, Liu Q, Zhou ZX, Zhao P, Wang GQ, Cai JQ. A nomogram to predict lymph node metastasis in patients with early gastric cancer. Oncotarget. 2017; 8:12203-12210.

5. Ronellenfitsch U, Lippert C, Grobholz R, Lang S, Post $\mathrm{S}$, Kahler G, Gaiser T. Histology-based prediction of lymph node metastases in early gastric cancer as decision guidance for endoscopic resection. Oncotarget. 2016; 7:10676-10683.

6. Gotoda T, Yanagisawa A, Sasako M, Ono H, Nakanishi Y, Shimoda T, Kato Y. Incidence of lymph node metastasis from early gastric cancer: estimation with a large number of cases at two large centers. Gastric Cancer. 2000; 3:219-225.

7. Hirasawa T, Gotoda T, Miyata S, Kato Y, Shimoda T, Taniguchi H, Fujisaki J, Sano T, Yamaguchi T. Incidence of lymph node metastasis and the feasibility of endoscopic resection for undifferentiated-type early gastric cancer. Gastric Cancer. 2009; 12:148-152.

8. Park SW, Lee H, Park CH, Jang HJ, Ahn H. Endoscopic and oncologic outcomes according to indication criteria of endoscopic resection for early gastric cancer: a systematic review and meta-analysis. Surgical Endoscopy and Other Interventional Techniques. 2016; 30:1270-1281.

9. Park CH, Shin S, Park JC, Shin SK, Lee SK, Lee YC, Lee H. Long-term outcome of early gastric cancer after endoscopic submucosal dissection: Expanded indication is comparable to absolute indication. Digestive and Liver Disease. 2013; 45:651-656.

10. Lee H, Yun WK, Min BH, Lee JH, Rhee PL, Kim KM, Rhee JC, Kim JJ. A feasibility study on the expanded indication for endoscopic submucosal dissection of early gastric cancer. Surgical Endoscopy and Other Interventional Techniques. 2011; 25:1985-1993.

11. Ahn JY, Jung HY, Choi KD, Choi JY, Kim MY, Lee JH, Choi KS, Kim DH, Song HJ, Lee GH, Kim JH, Park YS. Endoscopic and oncologic outcomes after endoscopic resection for early gastric cancer: 1370 cases of absolute and extended indications. Gastrointestinal Endoscopy. 2011; 74:485-493.

12. Japanese Gastric Cancer A. Japanese gastric cancer treatment guidelines 2010 (ver. 3). Gastric Cancer. 2011; 14:113-123.

13. Katai H, Sano T. Early gastric cancer: concepts, diagnosis, and management. Int J Clin Oncol. 2005; 10:375-383.

14. Catalano V, Labianca R, Beretta GD, Gatta G, de Braud F, Van Cutsem E. Gastric cancer. Crit Rev Oncol Hematol. 2009; 71:127-164.

15. Nishizawa T, Yahagi N. Long-Term Outcomes of Using Endoscopic Submucosal Dissection to Treat Early Gastric Cancer. Gut Liver. 2017.

16. Uedo N, Takeuchi Y, Ishihara R. Endoscopic management of early gastric cancer: endoscopic mucosal resection or endoscopic submucosal dissection: data from a Japanese high-volume center and literature review. Ann Gastroenterol. 2012; 25:281-290.

17. Gotoda T. Endoscopic resection of early gastric cancer. Gastric Cancer. 2007; 10:1-11.

18. Ono H. Early gastric cancer: diagnosis, pathology, treatment techniques and treatment outcomes. Eur J Gastroenterol Hepatol. 2006; 18:863-866.

19. Folli S, Morgagni P, Roviello F, De Manzoni G, Marrelli D, Saragoni L, Di Leo A, Gaudio M, Nanni O, Carli A, Cordiano C, Dell'Amore D, Vio A, et al. Risk factors for lymph node metastases and their prognostic significance in early gastric cancer (EGC) for the Italian Research Group for Gastric Cancer (IRGGC). Jpn J Clin Oncol. 2001; 31:495-499.

20. Park SW, Lee H, Park CH, Jang HJ, Ahn H. Endoscopic and oncologic outcomes according to indication criteria 
of endoscopic resection for early gastric cancer: a systematic review and meta-analysis. Surg Endosc. 2016; 30:1270-1281.

21. Ono H, Yao K, Fujishiro M, Oda I, Nimura S, Yahagi N, Iishi H, Oka M, Ajioka Y, Ichinose M, Matsui T. Guidelines for endoscopic submucosal dissection and endoscopic mucosal resection for early gastric cancer. Dig Endosc. 2016; 28:3-15.

22. Sekiguchi M, Oda I, Taniguchi H, Suzuki H, Morita S, Fukagawa T, Sekine S, Kushima R, Katai H. Risk stratification and predictive risk-scoring model for lymph node metastasis in early gastric cancer. J Gastroenterol. 2016; 51:961-970.

23. Zheng Z, Zhang Y, Zhang L, Li Z, Wu X, Liu Y, Bu Z, Ji J. A nomogram for predicting the likelihood of lymph node metastasis in early gastric patients. BMC Cancer. 2016; 16:92.

24. Ahmad R, Setia N, Schmidt BH, Hong TS, Wo JY, Kwak EL, Rattner DW, Lauwers GY, Mullen JT. Predictors of Lymph Node Metastasis in Western Early Gastric Cancer. J Gastrointest Surg. 2016; 20:531-538.

25. Shida A, Fujioka S, Kawamura M, Takahashi N, Ishibashi Y, Nakada K, Mitsumori N, Omura N, Yanaga K. Prediction of lymph node metastasis in patients with submucosa-invading early gastric cancer. Anticancer Res. 2014; 34:4471-4474.
26. Kim TJ, Lee H, Min YW, Min BH, Lee JH, Kim KM, Kim MJ, Kim K, Rhee PL, Kim JJ. One-dimensional and 2-dimensional tumor size measurement for prediction of lymph node metastasis in differentiated early gastric cancer with minute submucosal invasion. Gastrointest Endosc. 2017; 85:730-736.

27. The general rules for the gastric cancer study in surgery ad pathology. Part II. Histological classification of gastric cancer. Jpn J Surg. 1981; 11:140-145.

28. Japanese Gastric Cancer A. Japanese gastric cancer treatment guidelines 2014 (ver. 4). Gastric Cancer. 2017; 20:1-19.

29. del Casar JM, Corte MD, Alvarez A, Garcia I, Bongera M, Gonzalez LO, Garcia-Muniz JL, Allende MT, Astudillo A, Vizoso FJ. Lymphatic and/or blood vessel invasion in gastric cancer: relationship with clinicopathological parameters, biological factors and prognostic significance. J Cancer Res Clin Oncol. 2008; 134:153-161.

30. Rice TW, Blackstone EH, Rusch VW. 7th edition of the AJCC Cancer Staging Manual: esophagus and esophagogastric junction. Ann Surg Oncol. 2010; 17:1721-1724. 